\title{
Identification and Characterization of Previously Described Epitopes in HIV-1 Subtypes B, C, F and BF in Brazil
}

\begin{abstract}
Artur Trancoso Lopo de Queiroz ${ }^{1,2}$, Luciane Amorim Santos ${ }^{1,2}$, Domingos Ramon Moreau ${ }^{1}$, Tulio de Oliveira ${ }^{3}$, David I. Watkins ${ }^{4}$, Bernardo Galvão-Castro ${ }^{1,2}$ and Luiz Carlos Junior Alcantara ${ }^{1,2}$

${ }^{1}$ Advanced Public Health Laboratory, Gonçalo Moniz Research Center, Oswaldo Cruz Foundation, Salvador, BA, Brazil; ${ }^{2}$ Bahia School of Medicine and Public Health/Foundation for Development of Science, Salvador, BA, Brazil; ${ }^{3}$ Department of Zoology, University of Oxford, Oxford, United Kingdom; ${ }^{4}$ University of Wisconsin Medical School, Department of Pathology, Madison, USA
\end{abstract}

\begin{abstract}
Genetic analysis of HIV-1 is essential to improve treatment strategies and select epitopes for vaccine programs. The objective of this study was to determine whether known $\mathrm{CD}^{+}$and $\mathrm{CD8}^{+}$epitopes were present in Brazilian $\mathrm{HIV}-1$ strains. We used previously described $\mathrm{CD8}^{+}$and $\mathrm{CD4}^{+}$epitopes from the Los Alamos laboratory to search for these epitopes in the Brazilian sequences using the HIVbase program and we compared the frequency results with the analyses using physical-chemical profile tools from Network Protein Sequence Analysis (NPSA), and the SYFPEITHI program. Furthermore, this analysis was carried out with the Prosite tool using the GeneDoc program and ds/dn analyses using the Synonymous Nonsynonymous Analysis Program (SNAP). The HIVbase epitope mapping demonstrated that $30 \mathrm{CD8}^{+}$and $6 \mathrm{CD}^{+}$epitopes were present in the Brazilian sequences at a high frequency. Only two of these epitopes were heavily glycosylated. Interestingly, ds/dn analyses showed evidence of purifying selective pressure. These types of analyses could be useful for the assessment of possible vaccine efficiency in populations. Key-Words: HIV-1, env, vaccine, epitope.
\end{abstract}

HIV-1, identified as the etiological agent of AIDS [1], contributes to the development of immunodeficiency. Its biological complexity and high mutation rate have made the design of a vaccine to control the pandemic difficult. $\mathrm{CD} 8^{+} \mathrm{T}$ lymphocyte responses are largely responsible for controlling viral replication during both acute and chronic infection [2-4]. The antibody responses appears much later and select a mutant virus [5]. Mutations in CTL epitopes and sites recognized by antibodies and acquisition of glycosylation sites are escape mechanisms that allow the virus to replicate and infect more cells [5-7].

The mapping of epitopes in diverse virus proteins and the identification of possible modifications can provide useful information to aid vaccine development. For example, mutations in the TW10 and SL9 Gag epitopes result in a fitness cost to the virus [7], and this kind of information is very useful for the vaccine development process. The use of bioinformatics software can identify escaping epitopes. Bioinformatics programs have been developed for diverse areas including protein analysis, physical-chemical characteristics, MHC binding databases, and posttranslational modification. The use of these programs to study the molecular epidemiology and genetic variation of the HIV-1 epidemic in Brazil may provide important information Received on 27 October 2006; revised 22 January 2007.

Address for correspondence: Dr. Luiz Carlos J. Alcantara. Advanced Public Health Laboratory, Gonçalo Moniz Research Center, Oswaldo Cruz Foundation. Rua Waldemar Falcão 121, Candeal, Salvador, Bahia, Brazil. Zip code: 40296-610. Telephone \# 55713176 2246/3176 2213. Fax \# 55713176 2300. E-mail: lalcan@cpqgm.fiocruz.br. Financial Support: This project was partially supported by the Fundação de Amparo a Pesquisa do Estado da Bahia (FAPESB, grant number 303/ 03) and PN-DST/AIDS, Ministério da Saúde, Brasil (grant number 306 and 307/04).

The Brazilian Journal of Infectious Diseases 2007;11(1):27-30. (C) 2007 by The Brazilian Journal of Infectious Diseases and Contexto Publishing. All rights reserved. about the epidemic in this country. This information could be important in the design of an effective vaccine as well as for antiretroviral treatment. The objective of this study was to characterize genetic variation in previously identified epitopes in the Brazilian HIV-1 env sequences.

\section{Materials and Methods}

Sequences of all of the Brazilian HIV-1 strains $(3,813)$ were collected from GenBank and added to the HIVbase Database [8]. Epitope mapping analyses were performed as described in the HIV Immunology and HIV/SIV Los Alamos Vaccine Databases [9]. We selected and analyzed only the env region, consisting of 2,644 sequences from gp120: $\mathrm{C} 1 \mathrm{~V} 1=51 ; \mathrm{C} 2=59$; C3=200; V3loop=515; and gp41=50. The alignment of these sequences was carried out using the ClustalX [10] software. Genedoc [11] software was used to edit and translate the alignment, and the potential site analyses were performed using the Prosite [12] tool. For selective pressure analysis, we used the Synonymous Nonsynonymous Analysis Program (SNAP) [13] from Los Alamos. The proportion of synonymous substitutions per potential synonymous site and the proportion of nonsynonymous substitutions per potential nonsynonymous site were calculated using the Nei and Gojobori method [14]. Prediction of cellular epitopes was made with the SYFPEITHI online database [15]. Identification of Antibody epitopes and their physical-chemical characteristics were carried out using the Physico-chemical profiles program of the Network Protein Sequence Analysis (NPSA) [16-20].

\section{Results}

HIVbase epitope mapping showed that thirty $\mathrm{CD} 8^{+}$(Table 1) and six $C D 4^{+}$(Table 2) epitopes had a high frequency and showed varying degrees of conservation in the Brazilian HIV sequence. However, the SYPETEITHI analysis was restricted to the following HLA alleles: HLA-A*03, $-\mathrm{A} * 6801,-\mathrm{A} * 2402$ and $-A * 0201$. Two of the epitopes in this program had several 
Table 1. Mapping of the frequency of the Los Alamos CD8 epitopes in the Brazilian HIV-1 sequences

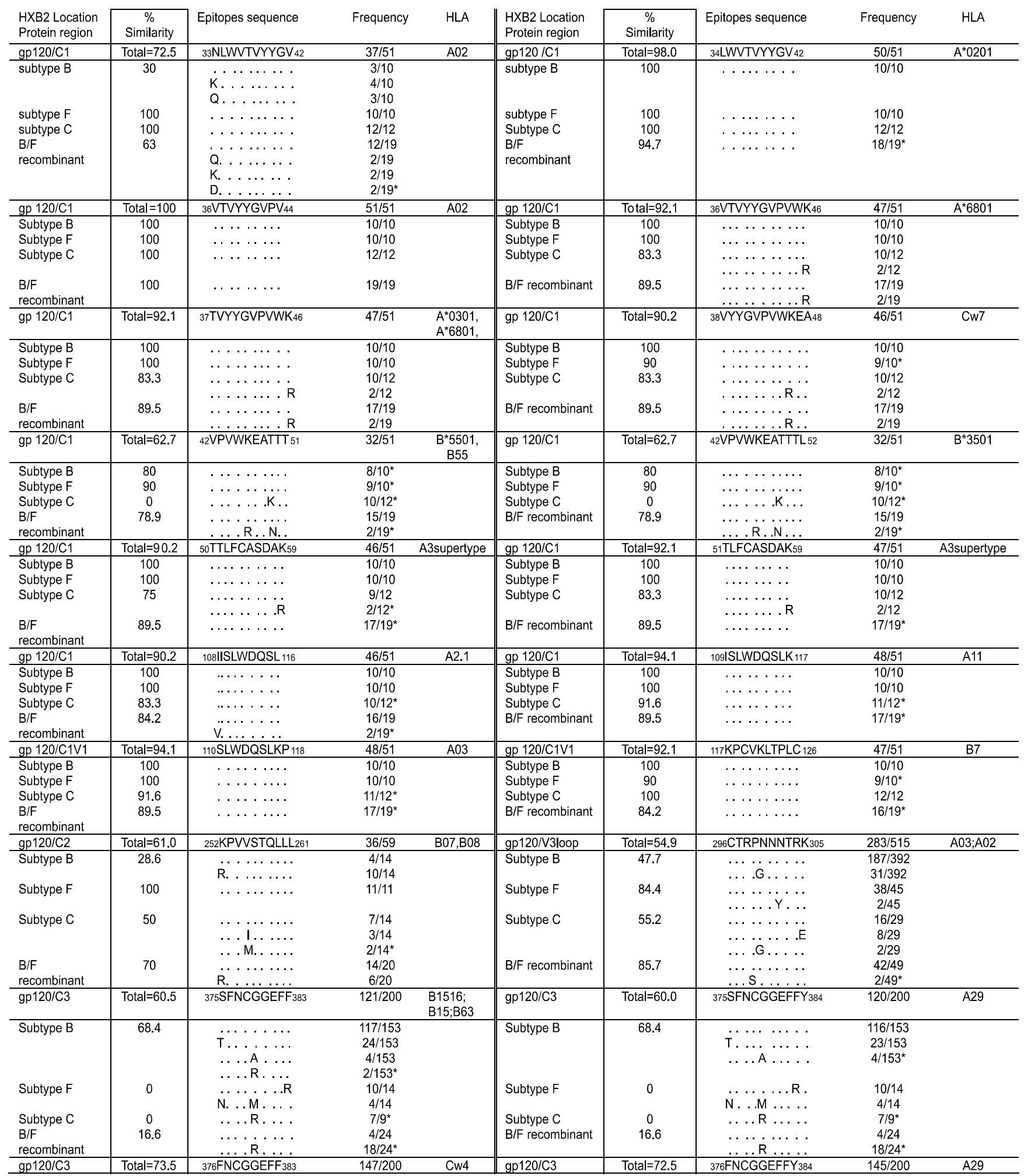


Table 1. (continued)

\begin{tabular}{|c|c|c|c|c|c|c|c|c|c|}
\hline Subtype B & 89.5 & $\begin{array}{l}\ldots \\
\ldots A \\
\ldots R\end{array}$ & $\begin{array}{c}141 / 153 \\
4 / 153 \\
2 / 153^{\star}\end{array}$ & & Subtype B & 88.6 & $A$ A & $\begin{array}{c}139 / 153 \\
4 / 153^{*}\end{array}$ & \\
\hline Subtype F & 0 & $\ldots M$ & $\begin{array}{c}10 / 14 \\
4 / 14\end{array}$ & & Subtype F & 0 & $\ldots \ldots R$ & $\begin{array}{c}10 / 14 \\
4 / 14\end{array}$ & \\
\hline Subtype C & 0 & $\ldots R \ldots$ & $7 / 9^{*}$ & & Subtype C & 0 & .R.... & $7 / 9^{*}$ & \\
\hline $\mathrm{B} / \mathrm{F}$ & 25 & $\cdots \ldots$ & $6 / 24$ & & $\mathrm{~B} / \mathrm{F}$ recombinant & 25 & $\ldots \ldots \ldots$ & $6 / 24$ & \\
\hline gp120/C3 & Total $=67.5$ & $\frac{\text {. . R.... }}{377 \text { NCGGEFFYCN } 386}$ & $\begin{array}{c}18 / 24 \\
135 / 200\end{array}$ & $N D^{* *}$ & an 11 & TTl-7 & $\ldots . R \ldots$ & $18 / 24$ & \\
\hline Subtype B & 88.6 & $\ldots \ldots \ldots$ & $129 / 153$ & & $\frac{\text { gp } 4 \perp}{\text { Subtyne } B}$ & $10 t a l=14.0$ & 529TMGAASITL537 & $37 / 50$ & $\mathrm{~A} 2$ \\
\hline & & $\ldots \ldots D$ & $\begin{array}{l}129 / 153 \\
10 / 153\end{array}$ & & subtype B & 28.6 & $\begin{array}{l}\ldots \ldots \ldots \\
\ldots \ldots \mathrm{L} .\end{array}$ & $\begin{array}{l}2 / 7 \\
2 / 7\end{array}$ & \\
\hline & & $\ldots A \ldots \ldots$ & $4 / 153^{*}$ & & & & . . . .VA. & $2 / 7^{*}$ & \\
\hline Subtype F & 0 & $\ldots \ldots$ R . & $9 / 14$ & & Subtype F & 90.9 & $\ldots \ldots \ldots$ & $10 / 11^{*}$ & \\
\hline & & $\ldots \mathrm{M} \ldots \ldots$ & $4 / 14^{*}$ & & & & & & \\
\hline Subtype C & 0 & $\ldots R \ldots \ldots$ & $7 / 9^{*}$ & & Subtype C & 100 & $\ldots \ldots \ldots$ & $13 / 13$ & \\
\hline $\begin{array}{l}\text { B/F } \\
\text { recombinant }\end{array}$ & 25 & $\begin{array}{l}\ldots \ldots \\
\ldots R\end{array}$ & $\begin{array}{c}6 / 24 \\
18 / 24\end{array}$ & & B/F recombinant & 63.2 & M. & $\begin{array}{l}12 / 19 \\
3 / 19^{*}\end{array}$ & \\
\hline gp 41 & Total $=52.0$ & 565LLQLTVWGI573 & $26 / 50$ & A2 & gp 41 & Total $=70.0$ & 584ERYLKDQQL592 & $35 / 50$ & B14,A32 \\
\hline Subtype B & 42.9 & $\ldots \ldots$ & $3 / 7$ & & Subtype B & 42.9 & $\ldots$ & $3 / 7$ & \\
\hline & & $\ldots \ldots . M$ & $4 / 7$ & & & & . . R . . . & $2 / 7$ & \\
\hline & & & & & & & $\ldots G \ldots$ & $2 / 7$ & \\
\hline Subtype F & 100 & $\cdots \cdots$ & $11 / 11$ & & Subtype F & 63.6 & $\ldots \ldots$ & 7/11 & \\
\hline Subtype C & 0 & M. . & $13 / 13$ & & Subtype C & 739 & $\cdots$ & $10 / 13$ & \\
\hline & & & & & & & $\ldots R$ & $2 / 13$ & \\
\hline & 63.2 & $\ldots$ & $12 / 19$ & & B/F recombinant & 78.9 & $\ldots \ldots \ldots$ & $15 / 19$ & \\
\hline recombinant & & & $6 / 19^{*}$ & & & & $\ldots Q \ldots$ & $3 / 19^{*}$ & \\
\hline gp 41 & Total $=70.0$ & 584ERYLKDQQLLG594 & $35 / 50$ & $\mathrm{ND}^{* *}$ & gp 41 & Total $=70.0$ & 585RYLKDQQLL593 & $35 / 50$ & $A^{*} 23, A 24$ \\
\hline Subtype B & 42.9 & $\ldots \ldots \ldots \ldots$ & $3 / 7$ & & Subtype B & 42.9 & $\ldots \ldots \ldots \ldots$ & $3 / 7$ & \\
\hline & & $\ldots R \ldots \ldots$ & $2 / 7$ & & & & $\ldots$ R. . . . & $2 / 7$ & \\
\hline & & $\ldots G \ldots \ldots$ & $2 / 7$ & & & & $\ldots$. . . . . . & $2 / 7$ & \\
\hline Subtype F & 63.6 & $\ldots \ldots$ & $7 / 11$ & & Subtype F & 63.6 & $\ldots \ldots \ldots$ & $7 / 11$ & \\
\hline & & $\ldots Q \ldots \ldots$ & $4 / 11$ & & & & $\ldots \mathrm{Q}$. & $4 / 11$ & \\
\hline Subtype C & 73.9 & $\ldots \ldots \ldots$ & $10 / 13$ & & Subtype C & 73.9 & $\ldots \ldots \ldots$ & $10 / 13$ & \\
\hline & & $\ldots R \ldots \ldots$ & $2 / 13^{*}$ & & & & $\ldots \mathrm{R}$. & $2 / 13$ * & \\
\hline $\mathrm{B} / \mathrm{F}$ & 78.9 & $\ldots \ldots \ldots \ldots$ & $15 / 19$ & & $\mathrm{~B} / \mathrm{F}$ recombinant & 78.9 & $\ldots \ldots \ldots$ & $15 / 19$ & \\
\hline recombinant & & $\ldots \mathrm{Q} \ldots \ldots$ & $3 / 19^{*}$ & & & & $\ldots \mathrm{Q} \ldots \ldots$ & $3 / 19^{*}$ & \\
\hline gp 41 & Total $=90.0$ & 678WLWYIKIFI686 & $45 / 50$ & A2 & gp 41 & Total $=82.0$ & 680WYIKIFIMl688 & $41 / 50$ & $A^{*} 2402$ \\
\hline Subtype B & 100 & $\ldots \ldots \ldots$ & $7 / 7$ & & Subtype B & 100 & $\ldots$ & $7 / 7$ & \\
\hline Subtype F & 90.9 & $\ldots \ldots \ldots$ & $10 / 11^{*}$ & & Subtype F & 90.9 & $\ldots \ldots \ldots$ & $10 / 11^{*}$ & \\
\hline Subtype C & 92.3 & $\ldots \ldots \ldots$ & $12 / 13^{*}$ & & Subtype C & 84.6 & $\ldots \ldots \ldots$ & $11 / 13^{*}$ & \\
\hline $\mathrm{B} / \mathrm{F}$ & 84.2 & $\ldots \ldots \ldots$ & $16 / 19$ & & B/F recombinant & 68.4 & $\ldots \ldots \ldots$ & $13 / 19$ & \\
\hline recombinant & & $\ldots$. R.. & $2 / 19^{*}$ & & & & .R..... & $2 / 19$ & \\
\hline & & & & & & & $\ddot{\mathrm{i}}$. & $2 / 19^{*}$ & \\
\hline gp 41 & Total $=82.0$ & 681 YIKIFIMIV689 & $41 / 50$ & A2 & gp 41 & Total $=60.0$ & 846RIRQGLERA859 & $30 / 50$ & $A^{*} 0205$ \\
\hline Subtype B & 100 & $\ldots \ldots \ldots$ & $7 / 7$ & & Subtype B & 100 & $\ldots \ldots \ldots$ & $7 / 7$ & \\
\hline Subtype F & 90.9 & $\ldots \ldots \ldots$ & $10 / 11^{*}$ & & Subtype F & 63.6 & ….... & $7 / 11$ & \\
\hline & & & & & & & $\ldots F$. F . & $4 / 11$ & \\
\hline Subtype C & 84.6 & $\ldots \ldots \ldots$ & $11 / 13^{*}$ & & Subtype C & 0 & $\ldots F \cdot A$ & $7 / 13$ & \\
\hline & & & & & & & $\ldots \ldots A$ & $3 / 13^{*}$ & \\
\hline $\mathrm{B} / \mathrm{F}$ & 68.4 & $\ldots \ldots \ldots$ & $13 / 19$ & & B/F recombinant & 84.2 & $\ldots \ldots \ldots$ & $16 / 19$ & \\
\hline recombinant & & .R... & $2 / 19$ & & & & $\ldots$ F. & $2 / 19^{*}$ & \\
\hline
\end{tabular}

* Mutation with frequency lower than $5 \%$ was excluded from the table. ${ }^{* *}$ HLA not determined. \% similarity: \% of sequences that have that epitope sequence. Frequency: $\mathrm{n}^{\circ}$ of sequences $/ \mathrm{n}^{\circ}$ of sequences that have that epitope or mutation.

Table 2. Mapping of the frequency of the Los Alamos CD4 epitopes in the Brazilian HIV-1 sequences

\begin{tabular}{|c|c|c|c|c|c|c|c|c|c|}
\hline $\begin{array}{l}\text { HXB2 Location } \\
\text { Protein region }\end{array}$ & $\begin{array}{c}\% \\
\text { Similarity }\end{array}$ & Epitope sequency & Frequency & HLA & $\begin{array}{l}\text { HXB2 } \\
\text { Location } \\
\text { Protein region }\end{array}$ & $\begin{array}{c}\% \\
\text { Similarity }\end{array}$ & Epitope sequency & Frequency & HLA \\
\hline gp 41 & Total $=50.0$ & 562QQHLLQLTVWGIKQL576 & $25 / 50$ & $N D^{\star \star}$ & gp $120 \mathrm{C} 1$ & Total $=88.2$ & 118 IISLWDQSLKPC 119 & $45 / 51$ & $N D^{* *}$ \\
\hline Subtype B & 42.8 & $\begin{array}{l}\ldots \ldots \ldots \ldots \\
\ldots \text { M. } \ldots \ldots \ldots \ldots\end{array}$ & $\begin{array}{l}3 / 7 \\
4 / 7\end{array}$ & & Subtype B & 100 & $\ldots \ldots \ldots$ & $10 / 10$ & \\
\hline Subtype F & 100 & $\ldots \ldots \ldots \ldots$ & $11 / 11$ & & Subtype F & 100 & $\ldots \ldots \ldots$ & $10 / 10$ & \\
\hline Subtype C & 0 & $\ldots$ M. ....... & $13 / 13$ & & Subtype C & 83.3 & $\ldots$ & $10 / 12^{\star}$ & \\
\hline $\mathrm{B} / \mathrm{F}$ recombinant & 57.9 & $\begin{array}{l}\ldots \ldots \\
\ldots M\end{array} \ldots \ldots$ & $\begin{array}{l}11 / 19 \\
6 / 19^{\star}\end{array}$ & & $\begin{array}{l}\text { B/F } \\
\text { recombinant }\end{array}$ & 78.9 & $\begin{array}{c}\ldots \ldots \ldots \\
v_{\ldots} \ldots \ldots \\
\ldots\end{array}$ & $\begin{array}{l}15 / 19 \\
2 / 19^{*}\end{array}$ & \\
\hline gp 41 & Total $=54.0$ & 593LGIWGCSGKLIC604 & $27 / 50$ & $\mathrm{ND}^{\star \star}$ & gp $120 \mathrm{C} 1$ & Total $=92.1$ & 110SLWDQSLKPCVKLTPL125 & $47 / 51$ & $\mathrm{ND}^{* *}$ \\
\hline Subtype B & 100 & $\ldots \ldots$ & $7 / 7$ & & Subtype B & 100 & $\ldots \ldots \ldots \ldots \ldots$ & $10 / 10$ & \\
\hline Subtype F & 0 & $\ldots L \ldots \ldots \ldots$ & $11 / 11$ & & Subtype F & 100 & $\ldots \ldots \ldots$ & $10 / 10$ & \\
\hline Subtype C & 100 & $\ldots \ldots \ldots \ldots$ & $13 / 13$ & & Subtype C & 91.6 & & $11 / 12^{*}$ & \\
\hline B/F recombinant & 36.8 & $\ldots \ldots \ldots$ & $7 / 19$ & & $\mathrm{~B} / \mathrm{F}$ & 84.2 & $\ldots \ldots \ldots \ldots$ & $16 / 19^{*}$ & \\
\hline & & $\begin{array}{l}\ldots \mathrm{L} \ldots \ldots \ldots \ldots \\
\ldots \mathrm{L} \ldots \ldots R .\end{array}$ & $\begin{array}{l}9 / 19 \\
2 / 19^{*}\end{array}$ & & recombinant & & & & \\
\hline gp 41 & Total $=54.0$ & 594GIWGCSGKLIC 604 & $27 / 50$ & $N D^{\star \star *}$ & gp 41 & Total $=54.0$ & 594GIWGCSGKLI603 & $27 / 50$ & $\mathrm{ND}^{\star *}$ \\
\hline Subtype B & 100 & $\ldots \ldots$ & $7 / 7$ & & Subtype B & 100 & & $7 / 7$ & \\
\hline Subtype F & 0 &.$L \ldots \ldots \ldots$ & $11 / 11$ & & Subtype F & 0 &. L. $\ldots \ldots \ldots$ & $11 / 11$ & \\
\hline Subtype C & 100 & $\ldots \ldots \ldots \ldots$ & $13 / 13$ & & Subtype C & 100 & $\ldots \ldots \ldots$ & $13 / 13$ & \\
\hline $\mathrm{B} / \mathrm{F}$ recombinant & 36.8 & $\ldots \ldots \ldots \ldots$ & $7 / 19$ & & $\mathrm{~B} / \mathrm{F}$ & 36.8 & $\ldots \ldots \ldots$ & $7 / 19$ & \\
\hline & &.$L \ldots \ldots \ldots$ & $9 / 19$ & & recombinant & & & $9 / 19$ & \\
\hline & & . L...R. . . & $2 / 19^{*}$ & & & & $\ldots \mathrm{R} \ldots$ & $2 / 19^{*}$ & \\
\hline
\end{tabular}

*Mutation with frequency lower than 5\% was excluded from the table. ** HLA not determined. \% similarity: \% of sequences that have the epitope. Frequency: $\mathrm{n}^{\circ}$ of sequences $/ \mathrm{n}^{\circ}$ of sequences that have the epitope or mutation. 
N-glycosylation sites (CTRPNNNTRK at amino acid position 296 to 305, at a frequency of 96.2\%, and NCGGEFFYCN at amino acid position 377 to 386 with a frequency of $84.5 \%$ ). The $d s / d n$ ratio was high in some of the $\mathrm{CD}^{+}$epitopes. This high ratio suggests that these particular epitopes may either not be under positive selection or are maintained under functional constraints.

The subtype B epitopes were the most conserved ones, especially in the $\mathrm{C} 1$ and $\mathrm{C} 3$ regions, followed by subtype $\mathrm{F}$. In the V3loop region, subtype $\mathrm{F}$ was the most conserved one. However, none of the most frequent mutations were associated with the loss of N-glycosylation site at this position. The gp41 was the most conserved region among the subtypes and this can be explained by the absence of variable regions in this protein. Epitopes in this region showed high $d s / d n$ ratios but these ratios were lower than the gp120 epitopes. This epitope conservation and the high $d s / d n$ ratio suggest that these regions may be important for viral fitness. Mutations in this region might change the protein structure, reducing the infection capacity of the virus.

The epitope VPVWKEATTTL is associated with a rapid progression HLA allele, HLA-B35 [4] and exhibited low variation in non-C subtypes. Interestingly, this epitope was highly variable in $\mathrm{B} / \mathrm{F}$ recombinants. This suggests that $\mathrm{CTL}$ may be exerting selective pressure on this subtype.

The regions involved in N-glycosylation were highly conserved. These sites are potentially important for the functioning of these proteins, and mutation in these regions might affect viral function.

\section{Conclusion}

An ideal vaccine would contain epitopes that would engender strong immune responses against the functionally important regions. Escape from these vaccine-induced immune responses would compromise viral fitness. Additionally, it will be important to continue analyzing epitope variability in other viral proteins in the HIV-1 strains circulating in Brazil as any eventual vaccine for use in Brazil will need to be relevant to the viruses in Brazil.

\section{Acknowledgements}

ATLQ and LAS contributed equally to this work. We are grateful to Elisabeth Deliege for her technical assistance.

\section{References}

1. Barré-Sinoussi F., Chermann J.C., Rey F., et al. Isolation of a Tlymphotropic retrovirus from a patient at risk for acquired immune deficiency syndrome (AIDS). Science 1983;220:868-71.
2. Matano T., Shibata R., Siemon C., et al. Administration of an anti-CD8 monoclonal antibody interferes with the clearance of chimeric simian/human immunodeficiency virus during primary infections of rhesus macaques. J Virol 1998; 72:164-9.

3. Jin X., Bauer D.E., Tuttleton S.E., et al. Dramatic rise in plasma viremia after CD8(+) $\mathrm{T}$ cell depletion in simian immunodeficiency virus-infected macaques. J Exp Med 1999;189:991-8.

4. Carrington M., Nelson G.W., Martin M.P., et al. HLA and HIV-1: heterozygote advantage and B35-Cw04 disadvantage. Science 1999;283:1748-52.

5. Burton D.R., Stanfield R.L., Wilson I.A. Antibody vs. HIV in a clash of evolutionary titans. Proc Natl Acad Sci 2005; 102:14943-8.

6. Reitter J.N., Means R.E., Desrosiers R.C. A role for carbohydrates in immune evasion in AIDS. Nat Med 1998;4:679-84.

7. Goulder P.J., Watkins D.I. HIV and SIV CTL escape: implications for vaccine design. Nat Rev Immunol 2004;4:630-40.

8. Lamers S., Beason S., Dunlap L., Compton R., Salemi M. HIVbase: a PC/Windows-based software offering storage and querying power for locally held HIV-1 genetic, experimental and clinical data. Bioinformatics 2004;20:436-8.

9. Bette T. M. Korber, Christian Brander, Barton F. Haynes, et al. HIV Immunology and HIV/SIV Vaccine Databases. Los Alamos National Laboratory, Theoretical Biology and Biophysics. Los Alamos, New Mexico. 2003.

10. Jeanmougin F., Thompson, J.D., Goy M., et al. Multiple sequence alignment with Clustal X. Trends Biochem Sci 1998;23:403-5.

11. Nicholas K.B., Nicholas H.B.J., Deerfield D.W. GeneDoc: Analysis and Visualization of Genetic Variation. EMBNEW.NEWS 1997; $4: 14$.

12. Falquet L., Pagni M., Bucher P., et al. The PROSITE database. Nucleic Acids Res 2002;30:235-8.

13. Korber B. HIV Signature and Sequence Variation Analysis. In: Allen G., Rodrigo, Learn G.H., eds. Computational Analysis of HIV Molecular Sequences. Dordrecht, Netherlands: Kluwer Academic Publishers, 2000, p.55-72.

14. Nei M., Gojobori T. Simple Methods for Estimating the Numbers of Synonymous and Nonsynonymous Nucleotide Substitutions. Mol Biol Evol 1986;5:418-26.

15. Rammensee H., Bachmann J., Emmerich N.P., et al. SYFPEITHI: database for MHC ligands and peptide motifs. Immunogenetics 1999;50:213-9.

16. Hopp T.P., Woods K.R. A computer program for predicting protein antigenic determinants. Mol Immunol 1983;20:483-9.

17. Kyte J., Doolittle R.F. A simple method for displaying the hydropathic character of a protein. J Mol Biol 1982; $157: 105-32$.

18. Karplus P.A., Schulz G.E. Prediction of chain flexibility in proteins. Naturwissens-chaften 1985;72:212-3.

19. Parker J.M., Guo D., Hodges R.S. New hydrophilicity scale derived from high-performance liquid chromatography peptide retention data: correlation of predicted surface residues with antigenicity and X-ray-derived accessible sites. Biochemistry 1986;25:5425-32.

20. Argos P., Rao J.K., Hargrave P.A. Structural prediction of membrane-bound proteins. Eur J Biochem 1982;128:565-75. 